\title{
Análise de material publicitário, disponível na internet, sobre analgésicos à base de dipirona que são vendidos sem a necessidade de prescrição, e os riscos da automedicação
}

\author{
Analysis of advertisement material available on the internet about dipyrone-based \\ analgesics that are sold without prescription, and the risks of self-medication
}

\author{
Lucas Curtolo Poiani ${ }^{\circledR 1}$, Charles Schmid ${ }^{\circledR 1}$, Luis Lopez Martinez ${ }^{\circledR 1}$
}

\section{Resumo}

Introdução: a regulamentação da propaganda de medicamentos no Brasil não é recente e atualmente temos a RDC 96 de 2008 da ANVISA que determina as regras das propagandas de medicamentos. Por conta da propagação da internet como forma de comunicação, as indústrias farmacêuticas vêm utilizando tal meio para divulgar informações sobre seus produtos. Tem sido mostrado que um dos princípios ativos mais associados a automedicação é a dipirona, sendo que os medicamentos com esse princípio ativo são os mais vendidos no país. Com isso, traz-se a questão de como essa forma de propaganda utilizando a internet tem influenciado a automedicação, e consequentemente, como o uso de medicamentos sem a orientação médica pode trazer riscos. Objetivo: análise do conteúdo da propaganda de medicamentos à base de dipirona na internet e sua possível influência na automedicação. Método: a dipirona (metamizol) foi escolhida como medicamento alvo desta pesquisa utilizando-se a lista dos 20 medicamentos mais vendidos no Brasil. Foram escolhidas as peças publicitárias a serem analisadas, utilizando-se a lista de medicamentos comercializados que possui dipirona como princípio ativo disponibilizada pela ANVISA. Foram consideradas páginas da internet e redes sociais e os materiais encontrados foram analisados com base na RDC 96 de 2008 da ANVISA, que determina as regras das propagandas de medicamentos. Foram excluídos desse estudo anúncios em perfis não oficiais das indústrias farmacêuticas e em endereços eletrônicos de drogarias. Resultados e Discussão: entre os medicamentos que possuíam páginas na internet ou redes

1. Faculdade de Ciências Médicas da Santa Casa de São Paulo. Curso de Pós-Graduação Lato Sensu em Pesquisa Clínica e Medical Affairs. São Paulo-SP - Brasil

Trabalho realizado: Faculdade de Ciências Médicas da Santa Casa de São Paulo. Curso de Pós-Graduação Lato Sensu em Pesquisa Clínica e Medical Affairs. São Paulo - SP - Brasil

Endereço para correspondência: Lucas Curtolo Poiani. Rua Miquelina 530, Apto. 3-09230-280 - Santo André-SP - Brasil sociais, foram observados que os principais pontos de atenção, à luz das normas aplicáveis ao tema, incluem: falta de informação suficientemente adequada sobre os riscos do uso do medicamento ou pouco destaque a essa informação; não destaque aos nomes dos princípios ativos; possível sugestão de diagnóstico e utilização de pessoas leigas indicando o uso do medicamento. Conclusão: dos materiais coletados, nota-se que a maioria deles não apresenta adequadamente os riscos e advertências associados ao uso do medicamento. Alguns outros pontos sensíveis encontrados são: suposta sugestão de diagnóstico e uso de pessoas reconhecidas por sua fama indicando a utilização do medicamento. Ao mostrar excessivamente os benefícios do medicamento, sem alertar proporcionalmente sobre os riscos associados, tais comunicações podem induzir os consumidores a riscos de saúde e estimular a automedicação. Por outro lado, notamos um constante aprimoramento das empresas anunciantes na tentativa de adequação de suas propagandas as resoluções aplicáveis, bem como, aos novos canais de comunicação. Neste sentido, acreditamos que uma atualização e simplificação das Resoluções que regulamentam o tema, torna-se necessária para sua melhor aplicação e fiscalização prática no cumprimento de seu legitimo objetivo que se concentra na proteção dos pacientes e consumidores destes produtos.

Palavras chave: Propaganda, Automedicação, Internet, Dipirona, Reações adversas a medicamentos

\begin{abstract}
Introduction: the regulation of drug advertising in Brazil is not recent and currently we have RDC 96 of 2008 from ANVISA that determines the rules for drug advertising. Because of the spread of the internet as a form of communication, pharmaceutical companies have been using this medium to disseminate information about their products. It has been shown that one of the active ingredients most associated with self-medication is dipyrone, and the drugs with this active ingredient are the most sold in the country. With this, the question arises as to how this form of adver-
\end{abstract}


Poiani LC, Schmid C, Martinez LL. Análise de material publicitário, disponível na internet, sobre analgésicos à base de dipirona que são vendidos sem a necessidade de prescrição, e os riscos da automedicação. Arq Med Hosp Fac Cienc Med Santa Casa São Paulo. 2020;65:e6.

tising using the internet has influenced self-medication, and consequently how the use of drugs without medical guidance can bring risks. Objective: content analysis of dipyrone-based drug advertising on the internet and its possible influence on self-medication. Methods: dipyrone (metamizole) was chosen as the target drug for this research using the list of the 20 most selling drugs in Brazil. The advertising pieces to be analyzed were chosen, using the list of commercialized drugs made available by ANVISA. The advertising pieces to be analyzed were chosen, using the list of commercialized drugs that have dipyrone as an active principle provided by ANVISA. internet pages and social media were considered, and the materials found were analyzed based on RDC 96 of 2008 by ANVISA, which determines the rules for advertising medicines. Excluded from this study were advertisements on unofficial profiles of the pharmaceutical industries and on drugstore e-mail addresses. Results and Discussion: Among the medicines that had pages on the internet or social networks, it was observed that the main points of attention, in the light of the rules applicable to the theme, include: lack of sufficiently adequate information on the risks of using the medicine or little emphasis on this information; do not highlight the names of the active ingredients; possible suggestion of diagnosis and use of lay persons indicating the use of the medicine. Conclusion: of the materials collected, most of them do not adequately present the risks and warnings associated with the use of the drug. Some other sensitive points found are: alleged suggestion of diagnosis and use of people recognized by their fame indicating the use of the drug. By showing excessively the benefits of the drug, without proportional warning about the risks associated, such communications can induce consumers to health risks and encourage self-medication. On the other hand, we noticed a constant improvement of the advertising companies in the attempt to adapt their advertisements to the applicable resolutions, as well as to the new communication channels. In this sense, we believe that an update and simplification of the Resolutions that regulate the theme became necessary for its better application and practical supervision in the fulfillment of its legitimate objective that focuses on the protection of patients and consumers of these products.

Keywords: Propaganda, Self medication, Internet, Dipyrone, Adverse drug reactions

\section{Introdução}

A propaganda de medicamento existe há tempos no país e registros mostram que já haviam formas de anúncio de produtos para saúde na época do império $^{(1)}$. Desde aquela época até a atualidade tal ferramenta vem sendo utilizada pela indústria farmacêutica como meio de divulgar os benefícios e promover a prescrição pelos profissionais prescritores ${ }^{(2)}$. Acompa- nhando essa tendência, autoridades implementaram formas de controle das informações veiculadas em tais propagandas ${ }^{(3)}$. Estudos mostram que tais materiais promovem em destaque os benefícios frente aos riscos associados ao uso dos medicamentos ${ }^{(4)}$, e dessa forma podem estimular o uso excessivo de medicamentos e automedicação ${ }^{(5-6)}$, causando principalmente uma preocupação com o aumento de eventos adversos pelo uso incorreto de medicamentos ${ }^{(7)}$.

A internet vem sendo utilizada como ferramenta de divulgação de produtos e serviços, incluindo medicamentos. Segundo o IBGE (Instituto Brasileiro de Geografia e Estatística) o país possui mais de 120 milhões de usuários de internet acima de $10 \operatorname{anos}^{(8)} \mathrm{e}$ associado a esse dado, um estudo transversal, quantitativo, no município de Crato, Ceará, Brasil, mostrou dentre a população que pratica a automedicação em um município de médio porte $(67,65 \%)$, a internet, como fonte de informação foi o meio de comunicação mais predominante ${ }^{(9)}$.

A automedicação, prática definida pela Organização Mundial da Saúde (OMS) como o uso de medicamentos por indivíduos para tratar de doenças ou sintomas detectados por si próprios ${ }^{(10)}$, pode trazer riscos à saúde ${ }^{(11)}$. Estudos mostram que no Brasil. a prevalência da automedicação atingiu 16\%, sendo a classe mais utilizada nessa prática foram os analgési$\cos ^{(12-13)}$, medicamentos esses vendidos sem prescrição médica ${ }^{(11-12)}$.

A dipirona, apontado como o princípio ativo mais consumido na automedicação ${ }^{(12)}$ e também como o mais vendido em 2017, segundo a Associação da Industria Farmacêutica e Pesquisa (Interfarma) $)^{(14)}$, além de muito consumido em associação com outros medicamentos, pode trazer riscos como: aumento da reação plaquetária( ${ }^{(15-16)}$, lesão renal, intoxicação por lítio e diminuição do efeito de anti-inflamatórios e beta-bloqueadores, pela semelhança com anti-inflamatórios não esteroidais (AINES) ${ }^{(16)}$.

Atualmente, no Brasil, as indústrias farmacêuticas devem seguir as exigências da Resolução da Diretoria Colegiada da ANVISA (RDC) 96 de 2008. Essa resolução traz as orientações para elaborar materiais promocionais de medicamentos tanto restritos à profissionais da saúde com também os disponíveis para o público em geral ${ }^{(17)}$.

De acordo com tal resolução, as indústrias detentoras dos registros de medicamentos devem promover informações comprovadas cientificamente e apresentar os riscos e advertências associadas ao uso dos medicamentos. Vale ressaltar que nesse item a resolução traz uma exigência para anúncios disponíveis na internet, determinando que informações sobre os riscos e advertências devem estar visíveis durante todo o tempo de navegação ${ }^{(17)}$. Tais propagandas não devem 
Poiani LC, Schmid C, Martinez LL. Análise de material publicitário, disponível na internet, sobre analgésicos à base de dipirona que são vendidos sem a necessidade de prescrição, e os riscos da automedicação. Arq Med Hosp Fac Cienc Med Santa Casa São Paulo. 2020;65:e6.

estimular o diagnóstico, e o uso indiscriminado de medicamentos através do uso de termos no imperativo tais como "use" ou "prescreva". Além disso, de acordo com a RDC 96 de 2008 da ANVISA que determina as regras das propagandas de medicamentos, tais materiais não devem utilizar imagens anatômicas, a menos que sejam ilustrativas ao tratamento e restringe o uso de personalidades reconhecidas pela fama que sejam leigas em farmácia e medicina.

\section{Objetivo}

O objetivo deste trabalho foi a análise do conteúdo da propaganda de medicamentos à base de dipirona na internet, que são vendidos sem a necessidade de prescrição, e se esse material estava de acordo com a RDC 96 de 2008 da ANVISA, que determina as regras das propagandas de medicamentos. Além disso, foi avaliada a possível influência desse material na automedicação.

\section{Métodos}

A dipirona (metamizol) foi escolhida como medicamento alvo desta pesquisa. Para essa escolha, utilizamos a lista dos 20 medicamentos mais vendidos no Brasil, disponibilizada pela Interfarma ${ }^{(14) .}$

Para escolhermos as peças publicitárias a serem analisadas por esta pesquisa, utilizamos a lista de medicamentos comercializados à base de dipirona, disponibilizada pela ANVISA ${ }^{(19)}$.

Realizamos uma busca, através da internet, das peças publicitárias dos medicamentos cujo princípio ativo fosse a dipirona (metamizol). Para isso, foram consideradas páginas da internet e redes sociais. Os materiais encontrados foram analisados com base na RDC 96 de 2008 da ANVISA, que determina as regras das propagandas de medicamentos.

A amostra selecionada constituiu em qualquer peça publicitária ou anúncio de analgésicos à base de dipirona (metamizol) encontrados na internet (redes sociais ou sites) disponíveis para o público em geral, ou seja, não restrito aos profissionais da saúde. Foram excluídos desse estudo materiais em perfis não oficiais das indústrias farmacêuticas e em endereços eletrônicos de drogarias.

\section{Resultados e Discussão}

\section{Páginas da internet}

Dos materiais encontrados na internet dez $(n=10)$ medicamentos possuem endereços eletrônicos onde os consumidores podem encontrar informações sobre o produto. As visitas a tais páginas da internet foram feitas entre os dias 05 e 11 de outubro de 2019. Abaixo listamos os pontos sensíveis de acordo com as normas aplicáveis no momento da visita. Os pontos encontrados que necessitam atenção, assim como, os pontos que estão de acordo com a RDC 96 de 2008 da ANVISA, estão apresentados nas tabelas 1 e 2 respectivamente.

Pela RDC 96 de 2008 da ANVISA, em materiais de produtos medicamentosos isentos de prescrição, se divulgados na internet, as informações sobre os riscos e advertências devem estar visíveis na página durante todo o tempo de navegação, e estar em fundo de retângulo branco, com letras pretas Humanist 777 Bold ou Frutiger 55 Bold em caixa alta.

Dentre os achados nos materiais analisados, dez $(n=10)$ estão relacionados à falta de informações claras sobre os riscos e as advertências do produto, seja pela ausência delas ou pela não adequação na forma de apresentar tais informações. Esse fato é relevante e preocupante considerando-se que em situações onde os materiais publicitários não dão a devida atenção aos riscos e advertências relacionadas aos produtos, podem expor os consumidores a riscos de saúde, pois, não deixam claro possíveis interações medicamentosas e eventos adversos. Além disso, a falta de informação clara e completa sobre o produto coloca tais propagandas em desarmonia com o previsto no Código de Defesa do Consumidor ${ }^{(18)}$ (Tabela 1).

\section{Tabela 1}

Número de peças publicitárias de medicamentos à base de dipirona disponíveis em páginas da internet que possuem pontos em desarmonia com a RDC 96 de 2008 da ANVISA

\begin{tabular}{cl}
$\begin{array}{c}\text { Número }(n) \text { de } \\
\text { peças publicitárias } \\
\text { ou anúncios } \\
\text { encontrados } \\
\text { em páginas da } \\
\text { internet }\end{array}$ & $\begin{array}{c}\text { Pontos importantes que estão em } \\
\text { desarmonia com a RDC 96 de } 2008 \text { da } \\
\text { ANVISA }\end{array}$ \\
\hline 10 & $\begin{array}{l}\text { Informações sobre riscos e advertên- } \\
\text { cias incompletas ou faltantes }\end{array}$ \\
\hline 3 & Sugestão de diagnóstico \\
\hline 2 & $\begin{array}{l}\text { Tamanho e disposição dos nomes dos } \\
\text { princípios ativos }\end{array}$ \\
\hline 1 & $\begin{array}{l}\text { Afirmação sobre comprovação cientí- } \\
\text { fica para público leigo }\end{array}$ \\
\hline 1 & Uso de termos no imperativo \\
\hline 1 & $\begin{array}{l}\text { Presença de pessoas sabidamente } \\
\text { leigas indicando o uso }\end{array}$ \\
\hline
\end{tabular}

Número de peças publicitárias de medicamentos à base de dipirona disponíveis páginas da internet que possuem pontos de desarmonia com a RDC 96 de 2008 da ANVISA (total de peças publicitárias analisadas: 10$)^{(17)}$ 
Poiani LC, Schmid C, Martinez LL. Análise de material publicitário, disponível na internet, sobre analgésicos à base de dipirona que são vendidos sem a necessidade de prescrição, e os riscos da automedicação. Arq Med Hosp Fac Cienc Med Santa Casa São Paulo. 2020;65:e6.

Foram encontrados três ( $\mathrm{n}=03)$ pontos de atenção onde os anúncios trazem possíveis causas das dores musculares, de barriga ou febre, sugerindo diagnósticos de doenças. Nesses pontos os anúncios descumprem a RDC 96 de 2008 da ANVISA, já que de acordo com a mesma, as propagandas de medicamentos não devem sugerir diagnóstico. Esse fato torna-se sensível uma vez que uma possível sugestão de diagnóstico no material publicitário pode induzir o paciente a não procurar orientação de um profissional para uma avaliação correta da causa da dor, fomentando a automedicação (Tabela 1).

Em dois $(n=02)$ materiais analisados os nomes dos princípios ativos não estavam apresentados de forma proporcional ao nome comercial do produto. Em alguns casos, os nomes comerciais e os princípios ativos estão presentes apenas nas imagens das embalagens. De acordo com a RDC 96 de 2008 da ANVISA, para produtos com apenas um princípio ativo, seu nome deve ser apresentado em no mínimo 50\% do tamanho do nome comercial. Para os medicamentos com mais de um princípio ativo estes devem ser apresentados em no mínimo 30\% do tamanho do nome comercial. Esse fato merece atenção, uma vez que, se o princípio ativo estiver presente de forma clara ao consumidor, permite que o mesmo tenha informações completas sobre o que está consumindo e esse direito lhe é garantido pelo Código de Defesa do Consumidor ${ }^{(18)}$ (Tabela 1).

Outros pontos sensíveis encontrados em menor frequência nas páginas da internet foram o uso de pessoa reconhecida pela fama, porém leiga em medicina ou farmácia, passando uma mensagem de possível indicação de uso do produto ou a utilização de mensagem imperativa, o que poderia levar ao uso indiscriminado do produto (Tabela 1).

A RDC 96 de 2008 da ANVISA por vezes apresenta orientações de difícil entendimento, pois proíbe que a informação sobre a eficácia do produto ser comprovada por estudos científicos esteja em materiais publicitários disponíveis para a população leiga, ao mesmo tempo, exige que quaisquer informações presentes em anúncios sejam baseadas em dados científicos. A presença de mensagem informando que a eficácia do produto foi comprovada por estudos científicos foi encontrada em um $(n=01)$ material direcionado ao público leigo, estando, portanto, em desarmonia com a RDC 96 de 2008 da ANVISA (Tabela 1).

Por outro lado, foram encontrados alguns pontos importantes que estão em harmonia com a RDC 96 de 2008 da ANVISA, que determina as regras das propagandas de medicamentos, tais como, em todas as páginas visitadas estavam presentes os dizeres "Se persistirem os sintomas o médico deverá ser consultado", além do fato de que em nenhuma página avaliada foi encontrada menções de que o medicamento em questão seria a única forma de tratamento (Tabela 2).

\section{Tabela 2}

Número de peças publicitárias de medicamentos à base de dipirona disponíveis em páginas da internet que possuem pontos em harmonia com a RDC 96 de 2008 da ANVISA

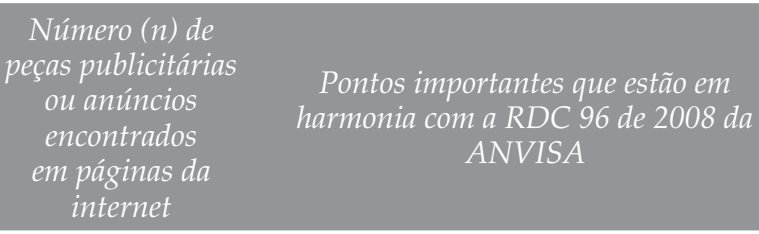

10

Dizeres "Se persistirem os sintomas, o médico deverá ser consultado"

10

Não houve menção de que o medicamento em questão era a única forma de tratamento

Número de peças publicitárias de medicamentos à base de dipirona disponíveis em páginas da internet que possuem pontos de harmonia com a RDC 96 de 2008 da ANVISA (total de peças publicitárias analisadas:10) $)^{(17)}$

\section{Redes Sociais}

Dos medicamentos com princípio ativo dipirona (metamizol), incluídos nesta pesquisa, com base na lista de medicamentos da ANVISA, dez $(n=10)$ apresentaram materiais disponíveis em redes sociais. As visitas nas redes sociais foram feitas entre os dias 12 de outubro e 27 de novembro de 2019. Os pontos encontrados que necessitam atenção, assim como, os pontos que estão de acordo com a RDC 96 de 2008 da ANVISA, estão apresentados nas tabelas 3 e 4 respectivamente.

Entre os achados nas redes sociais visitadas, podemos destacar principalmente a não adequação no fornecimento da informação dos riscos e advertências do uso dos produtos. Em 200 materiais encontrados nas redes sociais foram observados diversos pontos críticos sobre esse tema, onde observamos a ausência ou informação pouco clara sobre os riscos do produto, estando este fato em desarmonia com a RDC 96 de 2008 da ANVISA. A falta de informação suficientemente clara ao consumidor também se torna sensível à luz do Código de Defesa do Consumidor ${ }^{18}$. Além disso, o destaque dos benefícios do produto, sem a devida orientação clara e proporcional sobre os riscos e advertências do medicamento, pode trazer riscos aos pacientes por não contribuir para o uso racional de medicamentos e consequentemente automedicação (Tabela 3).

De acordo com a RDC 96 de 2008 da ANVISA, que determina as regras das propagandas de me- 
Tabela 3

Número de peças publicitárias de medicamentos à base de dipirona disponíveis nas redes sociais que possuem pontos de desarmonia com a RDC 96 de 2008 da ANVISA

\begin{tabular}{cl}
$\begin{array}{c}\text { Número }(n) \text { de } \\
\text { peças publicitárias } \\
\text { ou anúncios } \\
\begin{array}{c}\text { encontrados nas } \\
\text { redes sociais }\end{array}\end{array}$ & $\begin{array}{c}\text { Pontos importantes que estão em } \\
\text { desarmonia com a RDC } 96 \text { de } 2008 \text { da } \\
\text { ANVISA }\end{array}$ \\
\hline 200 & $\begin{array}{l}\text { Informações sobre riscos e advertên- } \\
\text { cias incompletas ou faltantes }\end{array}$ \\
\hline 1 & $\begin{array}{l}\text { Uso de imagens anatômicas sem fim } \\
\text { informativo para o tratamento }\end{array}$ \\
\hline 2 & $\begin{array}{l}\text { Presença de pessoas sabidamente } \\
\text { leigas indicando o uso }\end{array}$ \\
\hline 1 & $\begin{array}{l}\text { Clara menção do benefício do ufo, } \\
\text { frente ao não uso do medicamento }\end{array}$ \\
\hline 59 & $\begin{array}{l}\text { Sugestão de diagnóstico } \\
\text { Nomes dos princípios ativos meno- } \\
\text { res que 50\% do tamanho dos nomes } \\
\text { comerciais }\end{array}$ \\
\hline 15 & $\begin{array}{l}\text { Ausência dos nomes dos princípios } \\
\text { ativos }\end{array}$ \\
\hline
\end{tabular}

Nomes dos princípios ativos e comercial somente na embalagem do 58 produto* $^{*}$ Nesse último ponto, a RDC 96 de 2008 da ANVISA não deixa claro se isso seria uma forma de irregularidade.)

Número de peças publicitárias de medicamentos à base de dipirona disponíveis em redes sociais que possuem pontos de desarmonia com a RDC 96 de 2008 da ANVISA (total de peças publicitárias analisadas: 243$)^{(17)}$

dicamentos, em anúncios em páginas da internet as informações sobre os riscos e advertências devem estar visíveis na página durante todo o tempo de navegação, e estar em fundo de retângulo branco, com letras pretas Humanist 777 Bold ou Frutiger 55 Bold em caixa alta. Assim sendo, em uma $(n=01)$ oportunidade, foi observado que os riscos e advertências relacionados ao uso do medicamento em questão foram apresentados de forma clara ao consumidor, porém os mesmos não estavam totalmente de acordo com o formato determinado pela RDC 96 de 2008 da ANVISA (Tabela 4).

Outros pontos sensíveis encontrados em menor frequência nas redes sociais foram o uso de imagens anatômicas sem o intuito informativo sobre o tratamento $(n=01)$ o uso de pessoas leigas recomendando o uso do medicamento $(\mathrm{n}=02)$, a demonstração dos benefícios ao uso do medicamento frente ao não uso $(n=01)$, e a sugestão de possíveis causas de dores abdominais $(n=01)$, o que pode ser considerado uma
Tabela 4

Número de peças publicitárias de medicamentos à base de dipirona disponíveis nas redes que possuem pontos em harmonia com a RDC 96 de 2008 da ANVISA

\begin{tabular}{cl}
$\begin{array}{c}\text { Número }(n) \text { de } \\
\text { peças publicitárias } \\
\text { ou anúncios } \\
\begin{array}{c}\text { encontrados nas } \\
\text { redes sociais }\end{array}\end{array}$ & $\begin{array}{c}\text { Pontos importantes que estão em } \\
\text { harmonia com a RDC } 96 \text { de } 2008 \text { da }\end{array}$ \\
1 & $\begin{array}{l}\text { AnVISA } \\
\text { cias presentes de forma adequada }\end{array}$ \\
\hline 243 & $\begin{array}{l}\text { Não houve menção de que o medica- } \\
\text { mento em questão era a única forma } \\
\text { de tratamento }\end{array}$ \\
\hline 243 & Não uso de termos no imperativo \\
\hline
\end{tabular}

Número de peças publicitárias de medicamentos à base de dipirona disponíveis em redes sociais que possuem pontos de harmonia com a RDC 96 de 2008 da ANVISA (total de peças publicitárias analisadas: 243$)^{(17)}$

sugestão de diagnóstico em desarmonia com o que determina a RDC 96 de 2008 da ANVISA (Tabela 3).

Outro ponto importante a ser mencionado na análise da informação presente nas redes sociais é a não apresentação dos princípios ativos em tamanho proporcional ao tamanho do nome comercial. Nesse quesito, foram encontrados alguns pontos de atenção, seja presença dos princípios ativos em tamanho menor que $50 \%$ do nome comercial $(n=59)$, seja pela ausência dos princípios ativos (n-=15), ou pela presença apenas de imagens das embalagens contendo o nome comercial e os princípios ativos $(n=58)$. Nesse último ponto, a RDC 96 de 2008 da ANVISA não deixa claro se isso seria uma forma de irregularidade. Assim sendo, é evidente a necessidade de uma revisão e atualização das resoluções aplicáveis ao tema para atender a demanda destes novos canais de comunicação (Tabela 3).

Vale ressaltar que, apesar dos achados descritos anteriormente não seguirem todos os requisitos determinados pela RDC 96 de 2008 da ANVISA, por outro lado, em 243 publicações em redes sociais foram encontrados outros itens que atendem esta mesma resolução, tais como, ausência de indicações de que o medicamento em questão seria a única forma de tratamento ou ausência de linguagem imperativa (Tabela 4).

Além disso, notamos que algumas indústrias, em suas redes sociais, vêm apresentando tentativas de se adequarem à regulamentação vigente, no que diz respeito à divulgação das informações de segurança do produto na internet, seja pela adequação completa $(n=36)$ ou parcial da norma, aonde o quadro com as informações exigidas aparece apenas no final do anúncio $(\mathrm{n}=18)$ (Tabela 5). 
Tabela 5

Tentativa das indústrias farmacêuticas em adequarem as suas peças publicitárias à RDC 96 de 2008 da ANVISA no que diz respeito à apresentação de informações sobre a segurança do produto

\begin{tabular}{cl}
$\begin{array}{c}\text { Número }(n) \text { de } \\
\text { peças publicitárias } \\
\text { ou anúncios } \\
\text { encontrados nas } \\
\text { redes sociais }\end{array}$ & $\begin{array}{l}\text { Pontos importantes que inicialmente } \\
\text { estavam em desarmonia com a RDC } \\
96 \text { de } 2008 \text { da ANVISA e que foram } \\
\text { adequados pela indústria durante o } \\
\text { período desta pesquisa }\end{array}$ \\
\hline 36 & $\begin{array}{l}\text { Harmonização completa } \\
\text { Harmonização parcial (presença } \\
\text { das informações apenas ao final do } \\
\text { anúncio) }\end{array}$ \\
\hline
\end{tabular}

Número de peças publicitárias de medicamentos à base de dipirona disponíveis em redes sociais que apresentaram tentativas de adequação à RDC 96 de 2008 da ANVISA (total de peças publicitárias analisadas: 243$)^{(17)}$

\section{Conclusão}

Atualmente, no Brasil, as indústrias farmacêuticas devem seguir a RDC 96 de 2008 da ANVISA, que determina as regras das propagandas de medicamentos, incluindo normas para anúncios disponíveis na internet. Nesse trabalho, foi possível concluir que as indústrias de medicamentos estão cada vez mais presentes nos meios digitais, já que com o advento e popularização desse meio, o acesso da população à diversas informações e dados se tornou cada vez maior através dessa ferramenta de comunicação. Dos materiais coletados, nota-se que a maioria deles não apresenta adequadamente os riscos e advertências associados ao uso do medicamento. Alguns outros pontos sensíveis encontrados foram: suposta sugestão de diagnóstico e uso de pessoas reconhecidas por sua fama indicando a utilização do medicamento. Ao mostrar excessivamente os benefícios do medicamento, sem alertar proporcionalmente sobre os riscos associados, assim como, sobre os eventos adversos, tais comunicações podem induzir os consumidores a riscos de saúde que poderiam ser evitados com a correta orientação. Além disso, a forma na qual as propagandas de medicamentos são apresentadas na internet merece atenção quanto ao risco de estimular a automedicação, já que, em sua grande maioria, tais anúncios destacam muito mais as indicações e benefícios do medicamento e dão menor atenção e importância aos riscos e advertências pertinentes. Por outro lado, notamos um constante aprimoramento das empresas anunciantes na tentativa de adequação de suas propagandas às resoluções aplicáveis, bem como, aos novos canais de comunicação. Neste sentido, acreditamos que uma atualização e simplificação das Resoluções que regulamentam o tema, torna-se necessária para sua melhor aplicação e fiscalização prática no cumprimento de seu legitimo objetivo que se concentra na proteção dos pacientes e consumidores destes produtos.

\section{Referências}

1. Araújo CP, Bochner R, Nascimento ÁC. Marcos legais da propaganda de medicamentos: avanços e retrocessos. Physis. 2012 ; 22(1):331-46.

2. Reis CS, Moreira MZ, Murakami LC. Marketing de relacionamento na indústria farmacêutica como recurso para fidelização de clientes. Rev Gestão Conexões. 2018. 7(1):100-21.

3. Honorato F. Análise da propaganda de medicamentos em TV aberta para o Distrito Federal e "Entorno". Infarma. 2014; 26(1):35-44.

4. Abdalla MCE, Castilho SR. Análise da propaganda de medicamentos dirigida a profissionais de saúde. São Paulo. Rev Direiro Sanit. 2017; 18(1):101-20.

5. Fagundes MJD, Soares MGA, Diniz NM, Pires JR, Garrafa Vi. Análise bioética da propaganda e publicidade de medicamentos. Ciênc Saúde Coletiva. 2007; 12(1):221-9.

6. Luchessi AD, Marçal BF, Araújo GF, Uliana LZ, Rocha MRG, Pinto TJA. Monitoração de propaganda e publicidade de medicamentos: âmbito de São Paulo. Rev Bras Cienc Farm. 2005; 41(3):345-9.

7. Lima MG, Álvares J, Guerra Júnior AA, Guibu IA, Soeiro OM, Leite $\mathrm{SN}$, et al. Indicadores relacionados ao uso racional de medicamentos e seus fatores associados. Rev Saúde Pública. 2017; 51(Supl 2):23s.

8. Instituto Brasileiro de Geografia e Estatística (IBGE). PNAD Contínua TIC 2017: Internet chega a três em cada quatro domicílios do país. Agência IBGE Notícia. [Internet]. [citado 2019 Set 18]; 20/12/2018. Disponível em: https://agenciadenoticias.ibge. gov.br/agencia-sala-de-imprensa/2013-agencia-de-noticias / releases / 23445-pnad-continua-tic-2017-internet-chega-a-tres-em-cada-quatro-domicilios-do-pais

9. Gonçalves Júnior J, Moura ES, Dantas GCL, Lima AM, Brito WSB, Siebra BOB, et al. Influência da publicidade na automedicação na população de um município brasileiro de médio porte. J. Health Biol Sci (Online). [Internet]; 2018 [citado 2019 Set 18]; 6(2):152-5.

10. World Health Organization (WHO). Essential medicines and health products information portal. the benefits and risks of self-medication. Geneva: WHO; 2000. (Who Drug Information vol. 14, n.1, 2000). [citado 2019 Jul 13]. Disponível em: http:/ / apps.who.int/medicinedocs/en/m/abstract/Js21959en/

11. Pio A. Medicamentos isentos de prescrição devem ser ingeridos com cautela. Remédios vendidos livremente em farmácias em todo o mundo, dependendo do princípio ativo, podem causar problemas como alergias, lesões hepáticas e gastrointestinais e até levar a morte. Jornal Saúde Plena. [Internet]. [citado 2019 Set 17]; 20/04/2014. Disponível em: https://www.uai.com. br/app/noticia/saude/2014/04/20/noticias-saude,192564/ medicamentos-isentos-de-prescricao-devem-ser-ingeridos-com-cautela.shtml

12. Arrais PSD, Galvão TF, Andrade KRC, Araújo PC, Silva MT, Pereira MG. Prevalência da automedicação no Brasil e fatores associados. Rev Saúde Pública. 2016; 50(supl 2):13s.

13. da Silva Dal Pizzol T, Turmina Fontanella A, Cardoso Ferreira $\mathrm{MB}$, Bertoldi AD, Boff Borges R, Serrate Mengue S. Analgesic use among the Brazilian population: Results from the National Survey on Access, Use and Promotion of Rational Use of Medicines (PNAUM). PLoS One. 2019; 14(3):e0214329.

14. Interfarma. Associação da Indústria Farmacêutica de Pesquisa. Guia Inferfarma 2018. Ranking dos medicamentos. Ranking dos medicamentos mais vendidos - PPP*. [Internet]. [citado 2019 
Jul 13]. Disponível em https://www.interfarma.org.br/guia/ guia-2018/ranking_dos_medicamentos

15. Hartinger J, Novotny R, Bilkova J, Kvasnicka T, Mitas P, Sima $\mathrm{M}$, et al. Role of dipyrone in the high on-treatment platelet reactivity amongst acetylsalicylic acid-treated patients undergoing peripheral artery revascularisation. Med Princ Pract. 2018; 27(4):356-61.

16. Cazacu I, Mogosan C, Loghin F. Safety issues of current analgesics: an update. Clujul Med. 2015;88(2):128-36.

17. Brasil. Agência Nacional de Vigilância Sanitária (ANVISA). Resolução Diretoria Colegiada (RDC) n. 96, de 17 de dezembro de 2008. Dispõe sobre a propaganda, publicidade, informação e outras práticas cujo objetivo seja a divulgação ou promoção comercial de medicamentos. [Internet]. Brasília (DF); 2008. [2019 Jul 19]. Disponível em: http://portal.ANVISA.gov.br/documents/33864/284972/Resolucao_96_2008_consolidada_final_ site_setembro2010.pdf/837c25a1-7f86-4d89-913e-0543241efe27
18. Brasil. Lei nº 8.078 , de 11 de setembro de 1990. Código de Defesa do Consumidor. Dispõe sobre a proteção do consumidor e dá outras providências. [Internet]. Brasília (DF); 1990. [citado 2020 Jan 22]. Disponível em: http:/ / www.planalto.gov.br/ccivil_03/ Leis/L8078.htm

19. Brasil. Agência Nacional de Vigilância Sanitária (ANVISA). Listas de preços de medicamentos. [Internet]. Brasília (DF); 2019. [Citado 2019 Jul 19]. Disponível em: http:/ / portal.anvisa.gov.br / documents/374947/2829072/LISTA+CONFORMIDADE_201910-01.pdf/95fbce86-2855-422a-8ce3-9913efe17c74.

Trabalho recebido: 06/03/2020

Trabalho aprovado: 02/07/2020

Trabalho publicado: 03/07/2020 